\title{
Mechanical features and in vivo imaging of a polymer stent
}

\author{
Willem J. van der Giessen, Cornelis J. Slager, Elma J. Gussenhoven, Heleen M.M. van Beusekom, \\ Ruud A. Huijts ${ }^{1}$, Johan C. Schuurbiers, Richard A. Wilson, Patrick W. Serruys \& Pieter D. Verdouw \\ Department of Cardiology, Thoraxcentre, Ee 2357, Erasmus University Rotterdam, P.O. Box 1738, 3000 DR \\ Rotterdam, The Netherlands; ${ }^{1}$ Akzo Research Laboratories, Arnhem, The Netherlands
}

Accepted 17 February 1993

Key words: polymer, PETP, stent, angiography, intravascular ultrasound, morphology

\begin{abstract}
A polyethylene-terephthalate (PETP, polyester), self-expanding, braided mesh stent has been developed for percutaneous (coronary) arterial implantation. In vitro measurements showed that the radial pressure delivered by this device was similar to a self-expanding, stainless steel stent. Due to hysteresis-like behaviour, it proved necessary to mount the polymer stent on the delivery system immediately before the placement procedure, and to select a diameter in the unconstrained condition, which was $60 \%$ larger than the diameter of the target vessel.

Eight polyester stents were implanted in peripheral arteries of four pigs. Except for heparin during the implantation procedure, antithrombotic or antiplatelet drugs were not administered. After four weeks repeat angiography revealed that one of the stents was subtotally occluded. At autopsy, two other stents proved to be located in the aortic bifurcation, probably due to failure of the delivery system. Quantitative angiographic assessment showed that the mean luminal diameters at the site of stent placement were $3.3 \pm 0.2 \mathrm{~mm}$ before, $3.2 \pm 0.2 \mathrm{~mm}$ immediately after, and $2.7 \pm 0.5 \mathrm{~mm}$ at four weeks after implantation. Intravascular ultrasound (IVUS) examination after 4 weeks could identify the individual struts of the stents, as well as their length. In addition, a description of the extent of neointimal hyperplasia was feasible. The IVUS assessment was validated by histological examination.

In conclusion, polyester stents can be constructed with mechanical properties similar to stainless steel stents. After implantation in porcine peripheral arteries, five of six correctly placed stents were patent at four weeks. Imaging of stents by angiography and IVUS provided complementary information.
\end{abstract}

\section{Introduction}

A novel approach for the treatment of acute complications and the prevention of restenosis after percutaneous transluminal angioplasty (PTA) is the placement of a metallic endovascular prosthesis (stent) [1]. Results from uncontrolled clinical studies with stainless steel stents in the coronary circulation indicate, however, that these devices are prone to (sub)-acute thrombotic occlusion and do not eli- minate the incidence of restenosis, although they reduce its occurrence [2-5]. In animal experiments coronary stents composed of tantalum proved to be less thrombogenic than stainless steel stents [6], but the initial results after implantation in patients showed no detectable advantage of tantalum [7]. Applying a polymer coating to metal stents proved effective in reducing the thrombogenicity of these devices in experimental animals [8]. A polymeric stent offers the advantage of more versatility in de- 

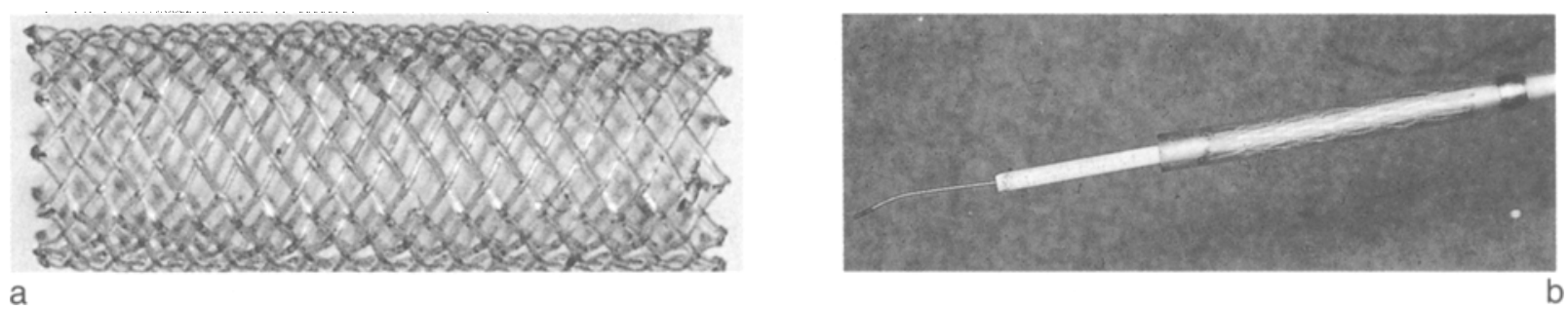

Fig. 1a. Mesh stent, braided of 24 polyester monofilaments, each $176 \mu \mathrm{m}$ in diameter. The unconstrained diameter of the stent is $5.3 \mathrm{~mm}$, and its length $15 \mathrm{~mm}$. Fig. $1 \mathrm{~b}$. Polyester mesh stent mounted on the coaxial delivery system. Withdrawal of the outer catheter of this system gradually releases the stent, which is prevented from withdrawal together with the outer catheter by the radiopaque stop.

signing mechanical properties. In addition, a high concentration of antithrombotic drugs, as well as agents mitigating the tissue response to the foreign material, can be contained in a polymer matrix [9]. In the present study, we report on the feasibility of placement and subsequent imaging of a stent composed entirely of synthetic polymer.

\section{Methods}

\section{Polymer stent}

The stent developed is a cylindrical braided mesh, constructed of 24 polyester (polyethylene-terephthalate, AKZO BV, Arnhem, The Netherlands) monofilaments of $176 \mu \mathrm{m}$ in diameter (Fig. 1a). The fibres at both ends of the stent were thermally fused. This stent can be constrained on a delivery system, consisting of two coaxial catheters (Fig. 1b). The diameter of the delivery catheter plus stent is $2.8 \mathrm{~mm}$. Withdrawal of the outer catheter will release the stent progressively, which anchors itself against the arterial wall, when the diameter of the stent after release exceeds the vessel diameter. The unconstrained diameter of the stents used in this study is $5.3 \mathrm{~mm}$.

\section{In vitro force - length measurements}

Long segments (>100 mm) of stent material were positioned in a force-length (F-L) measurement system. Both extremities of the stent had limited expansile properties, because they were fixed in the system, and therefore only the mid-section was used for measuring the F-L relationship. Data were obtained at $20^{\circ} \mathrm{C}$ and at $37^{\circ} \mathrm{C}$.

Using this F-L measurement system, the selected mid-segment of the stent was elongated by applying several known forces $(F)$. The maximal force $F$ was applied for 5 minutes and thereafter the force was gradually, decreased allowing the polyester stent to show its spring-like behaviour and gain in diameter again. Knowledge of the geometrically determined length $(\mathrm{L})$ - radius $(\mathrm{r})$ relationship $(\mathrm{L}=f(\mathrm{r}))$ and the surface area $(A=2 \pi . r . L)$ of this specific cylindric stent construction (Fig. 2), allowed the calculation of its radial pressure $(\mathrm{P})$ from the equation: P.A. $\Delta \mathrm{r}=-\mathrm{F} . \Delta \mathrm{L}$, assuming equal work to be delivered by either pressure $P$ or force $F$. The application of this formula yields global delivered pressure, assuming a stent with a closed cylindric surface. Correction for the actual fibre-covered area to total stent area allowed calculation of the locally delivered pressure at the fibre surface. Conversion of length to diameter was again calculated using the length-radius relationship.

\section{Stent expansion after release in vitro}

The polyester stent was constrained on its delivery system. After 15 minutes the stent was released and its diameter measured immediately, and again at 1 , 5,15 and 30 minutes and 15 hours using a stereomicroscope.

Similar diameter measurements were obtained for the Wallstent after release from its delivery system. 


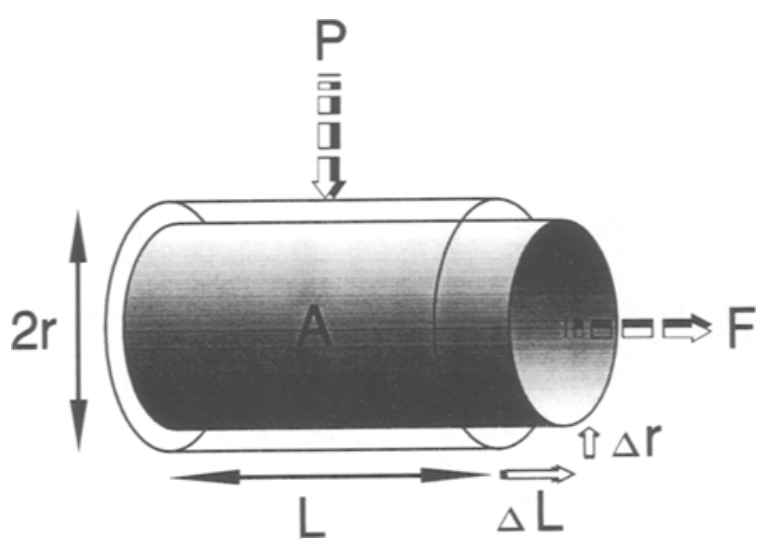

Fig. 2. Schematic drawing of two geometric conditions of the stent. The parameters used for the calculation of the pressurediameter relationship are explained in the text.

\section{In vivo stent implantation}

Experiments were performed in four Göttingen minipigs (24-28 kg). The protocol was approved by the Committee on experimental animals of the Erasmus University. After anaesthesia and under sterile conditions an arteriotomy of the left carotid artery was performed and a $9 \mathrm{~F}$ introduction sheath was placed as described earlier $[6,10]$. After administration of $5000 \mathrm{IU}$ of heparin sodium, an $8 \mathrm{~F}$ catheter was advanced to the infrarenal abdominal aorta. Isosorbide dinitrate was administered intra-arterially before angiography of the femoral arteries was performed. Iopamidol (Iopamiro ${ }^{\top M} 370$, Dagra BV, Diemen, The Netherlands) was used as contrast agent.

From the angiograms, a segment with a diameter of about $3.5 \mathrm{~mm}$ was selected. Thereafter, the stent delivery catheter was advanced over a 0.014 inch steerable guide wire to the site preselected for implantation, and the stents were placed.

After repeat angiography of the stented arteries, the introducer sheath was removed, the arteriotomy repaired and the skin closed in two layers. The animals were allowed to recover from anaesthesia, while post-procedure antithrombotic drugs were not administered.
Follow-up angiography and quantitative analysis

The catheterization procedure at 4 weeks follow-up was similar as described above. Angiography was performed in the same projection, and using identical settings of the X-ray equipment as during implantation. Angiograms (pre-implantation, immediately after implantation and after 4 weeks) were analyzed using the Cardiovascular Measuring System software (CMS) [11]. Mean luminal diameter at the site of stent placement was measured.

\section{Intravascular ultrasound}

At the time of repeat angiography the stented femoral arterial sites were studied using a mechanical intravascular ultrasound device [12]. The ultrasound catheter (5F, $30 \mathrm{MHz}$, Du-Med Rotterdam, The Netherlands) was introduced via the same $9 F$ introducer sheath as was used during the angiography. Under fluoroscopic guidance the catheter was advanced to the stent containing segment. The stent length was determined by marking the shaft of the catheter. The stent fibres and the neointimal hyperplasia at the luminal side of the stent fibres were assessed qualitatively.

\section{Histological measurements}

The abdominal aorta was cannulated and, after administration of a lethal dose of pentobarbital sodium, cross clamped proximal to the cannula. Through the cannula $500 \mathrm{ml}$ of saline followed by $500 \mathrm{ml}$ of buffered $4 \%$ paraformaldehyde was infused. The femoral arteries were dissected free and the stented segments and adjacent unstented segments were placed in $4 \%$ paraformaldehyde phosphate buffer ( $\mathrm{pH} 7.30$ ) for at least 48 hours in preparation for light microscopy, as described earlier [6, $10]$.

For the histological measurement of the thickness of the various layers of the arterial wall, at least two perpendicular resorcin-fuchsine stained sections of the proximal, middle and distal part of each stented arterial segment were observed by a micro- 
scope, connected to a videocamera, and displayed on a monitor. The external- and internal elastic lamina and the endothelial lining were traced using an integrated image analysis system (IBAS-2000, Kontron, Oberkochen, FRG) [13]. The distance between the endothelial lining and the internal elastic lamina or the stent fibres was taken as the thickness of the neointima. The media was defined as the layer between the internal- and external elastic lamina.

\section{Statistical analysis}

All quantitative data are expressed as mean \pm SEM. The significance of the changes in the angiographic data was evaluated by analysis of variance (ANOVA) and a two-sample t-test. The histological measurements were analyzed by the Wilcoxon Rank Sum test. A P value less than 0.05 was considered statistically significant.

\section{Results}

In vitro radial pressure-diameter relationship

Figure 3 illustrates that by reducing the diameter of the unconstrained Wallstent from 6.2 to $5.0 \mathrm{~mm}$, a global pressure of approximately $70 \mathrm{mmHg}$ is exerted. After correction for the actual fibre surface area, because the stent is not a closed cylinder but an open weave structure, this results in a local pressure of the individual wires of more than $400 \mathrm{mmHg}$. The polyester stent shows increases in radial pressure, after reduction of the diameters from 5.3 to $4.0 \mathrm{~mm}$, similar to those observed with the stainless steel stent. Measurements at $37^{\circ} \mathrm{C}$ and at $20^{\circ} \mathrm{C}$ gave identical results.

\section{Hysteresis-like behaviour after release in vitro}

Unlike the stainless-steel Wallstent, the self-expanding polyester stent did not return to its unconstrained diameter after release from the delivery catheter (Fig. 4). At 30 minutes and at 15 hours after

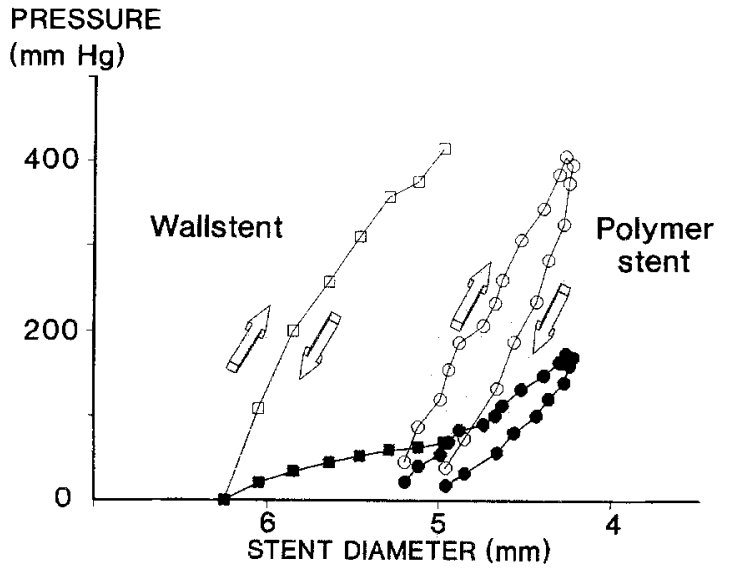

Fig. 3. Pressure-diameter relation of the stainless steel Wallstent (squares) and the polymer (polyethylene-terephthalate) stent (circles). Reducing the diameter of both stents results in an equivalent build-up of radial pressure assuming the stents are closed cylindrical structures ('global' pressure, closed symbols). Correcting for the open surface area of the stents results in even more impressive pressures at the wire surface (open symbols). The curve for the polymer stent shows hysteresis-like behaviour, as the pressures needed to constrain the diameter of the stent are considerably less after 5 minutes (downward pointing arrow). Data for the Wallstent prosthesis were published earlier [19].

release, the polyester stent had lost $7 \%$ of its unconstrained diameter.

\section{In vivo implantation}

Placement of all stents seemed easy at the preselected arterial sites. After 4 weeks one stented femoral artery proved subtotally occluded by angiography. An unexpected finding at autopsy was that in one animal both stents were found patent at the aortic bifurcation, extending into, but not occluding, the origin of both iliac arteries.

\section{Quantitative angiography}

The mean diameter at the site of the stents in the arteries with correctly placed stents measured before implantation $3.3 \pm 0.2 \mathrm{~mm}$ in diameter (Table 1). After implantation this value was $3.2 \pm 0.2 \mathrm{~mm}$, confirming that the stents did not overstretch the vessels. At four weeks the five patent arteries mea- 


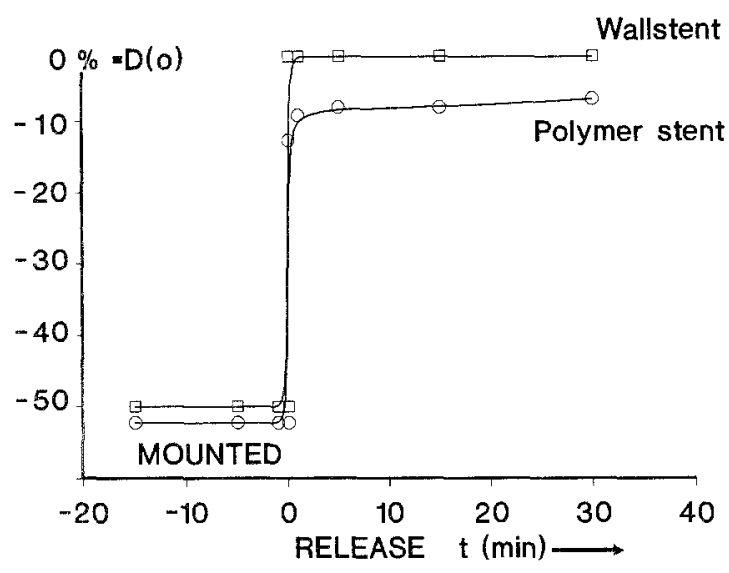

Fig. 4. Percentage change in stent diameter from unconstrained diameter $(0 \%)$ of the polyester stent, compared to the Wallstent. Mounting both types of stents on a delivery system reduces their diameter by $50 \%$. Subsequent release causes the Wallstent to regain its original diameter immediately. The polyester stent does not return to its original diameter within 30 minutes.

sured $3.1 \pm 0.3 \mathrm{~mm}$, while one stent was found subtotally occluded with a diameter of $0.8 \mathrm{~mm}$. The mean difference between the luminal diameters at four weeks and immediately after implantation was $0.5 \mathrm{~mm}$ (95\% confidence limits: $-0.54 \mathrm{~mm}$ and $+1.54 \mathrm{~mm}, \mathrm{P}>0.05)$.

\section{Intravascular ultrasound}

Using intravascular ultrasound the stents could be easily identified incorporated in the respective arteries. Individual struts of the stents were noted to be highly echoreflectant with or without reverberations beyond the stent (Fig. 5). The native femoral artery was recognized as a homogeneous bright arterial wall corresponding to the elastic nature of the vessel. The length of the endoprosthesis determined with the ultrasound device corresponded closely with the actual length of the stent in the excised histological specimen.

In addition, neointimal hyperplasia was recognized as the presence of a soft echostructure inside the lumen superimposed to the stent surface.
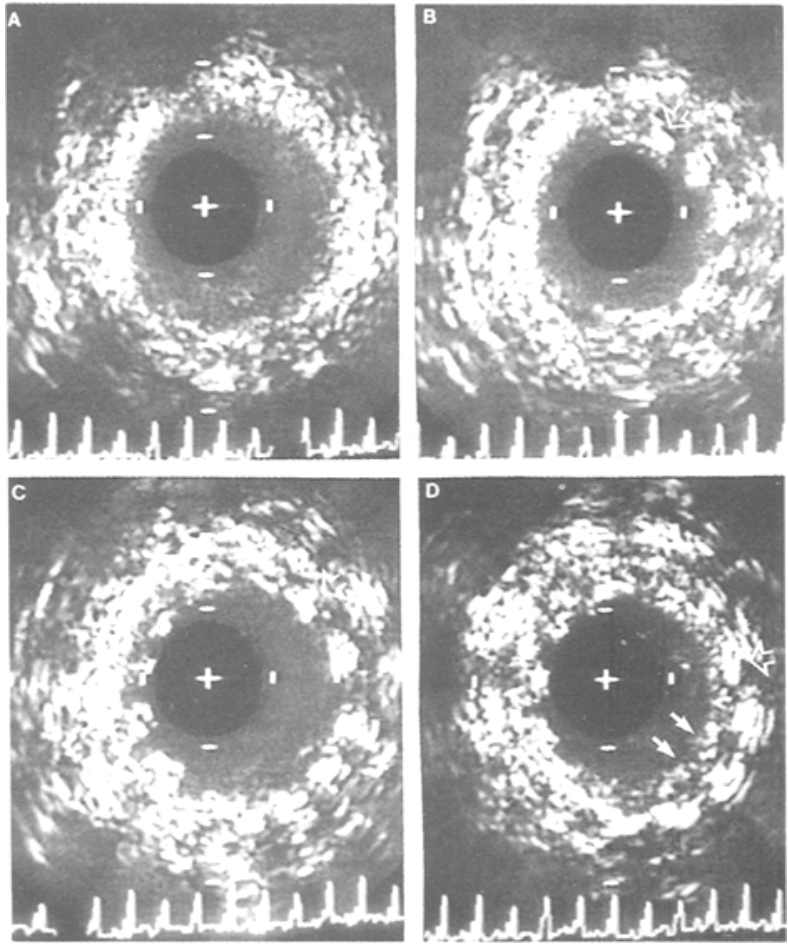

Fig. 5. Representative intravascular ultrasound images of the femoral artery and stent. Panel A shows the femoral artery recognized as a homogeneous echoreflectant structure. Panel B shows the junction of femoral artery to stent; the individual fibres are seen as highly bright echoes (open arrow in B, C and D). Differentiation can be made between the stent without (panel C) and with intimal hyperplasia (panel D, white, closed arrows).

Table 1. Individual and mean values of the average diameters of the stented arteries as determined by quantitative angiographic analysis.

\begin{tabular}{lllll}
\hline $\begin{array}{l}\text { Animal } \\
\text { number }\end{array}$ & Artery & $\begin{array}{l}\text { Pre-stent } \\
(\mathrm{mm})\end{array}$ & $\begin{array}{l}\text { Post-stent } \\
(\mathrm{mm})\end{array}$ & $\begin{array}{l}4 \text { weeks } \\
(\mathrm{mm})\end{array}$ \\
\hline 3368 & l femoral & 3.8 & 3.2 & 3.4 \\
3368 & 1 carotid & 3.9 & 3.5 & 4.0 \\
3378 & r fem & 3.0 & 3.3 & 2.7 \\
3378 & 1 fem & 2.9 & 3.3 & 2.6 \\
3391 & r fem & 2.8 & 3.6 & 2.9 \\
3391 & 1 fem & 3.3 & 2.5 & 0.8 \\
\multirow{2}{*}{ Mean \pm sem } & $3.3 \pm 0.2$ & $3.2 \pm 0.2$ & $2.7 \pm 0.5$ \\
\hline
\end{tabular}

$\mathrm{r} / \mathrm{l}$ fem $=$ right/left femoral artery $; \mathrm{l}$ carotid $=$ left carotid artery. 


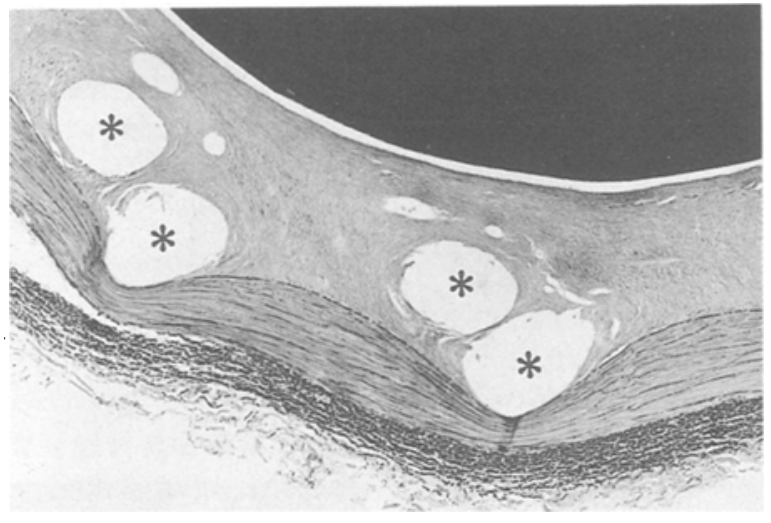

Fig. 6. Light microscopical picture of a porcine femoral artery four weeks after implantation of the polymer stent. During processing the polymer material has been removed. The voids formerly occupied by the stent fibres have been indicated $(*)$. The elastin stain (resorcin-fuchsin) shows elastin fibres in black. Clearly the impression of the media is shown at both fibre crossings. The neointima on top of the fibres has a limited thickness, and shows some neovascularizations indicating the organization. of thrombus.

\section{Histology}

Macroscopically the stents proved expanded as expected, and collapse or recoil was never observed. The luminal side was covered by a thin glistening membrane. Light microscopy revealed that the stent was completely embedded in the vascular wall. On top of the fibres a neointima consisting of smoot muscle cells in a collagenous matrix was observed, while a mild inflammatory reaction surrounded the fibres (Fig. 6). The media under the fibres was impressed by $27 \%$ (Table 2), but other abnormalities of the media were not observed. The neointima measured on top of the polymer filaments had a thickness of $114 \pm 38 \mu \mathrm{m}$ and between the fibres $246 \pm 44 \mu \mathrm{m}$ (Table 2). The media under the threads had a thickness of $132 \pm 41 \mu \mathrm{m}$, and between the threads $173 \pm 41 \mu \mathrm{m}(\mathrm{n}=5)$. The difference in thickness of both the neointima and the media at the two sites did not reach levels of statistical significance $(\mathrm{P}=0.09)$.

\section{Discussion}

\section{Choice of polymer and construction of stent}

Polyethylene-terephthalate is currently the most frequently used material for vascular grafts. It has considerable tensile strength and stiffness, and is easy to handle for construction or design purposes. The uptake of water is maximally $0.1 \%$, and does not influence mechanical behaviour or glass transition temperature of the polymer, an important quality of a material intended for implantation in vivo. The mechanical properties of (drawn) polyester fibres can be varied within considerable limits depending on processing conditions.

In vitro pressure-diameter relationship and hysteresis-like behaviour

The results of the in vitro measurements confirm

Table 2. Individual and averagee values of the thickness of the vessel wall layers as measured by morphometry.

\begin{tabular}{|c|c|c|c|c|c|c|}
\hline Animal & Artery & Neointima & Neointima stent & Media & Media stent & Medial impression \\
\hline 3368 & $\mathrm{r}$ fem & $133 \pm 15$ & $42 \pm 10$ & $131 \pm 24$ & $81 \pm 7$ & $38 \%$ \\
\hline 3368 & l car & $228 \pm 26$ & $78 \pm 13$ & $334 \pm 16$ & $296 \pm 18$ & $12 \%$ \\
\hline 3378 & $\mathrm{r}$ fem & $193 \pm 12$ & $51 \pm 5$ & $115 \pm 2$ & $90 \pm 5$ & $21 \%$ \\
\hline 3378 & 1 fem & $285 \pm 55$ & $150 \pm 28$ & $147 \pm 8$ & $105 \pm 10$ & $29 \%$ \\
\hline 3391 & $\mathrm{r}$ fem & $392 \pm 27$ & $246 \pm 17$ & $135 \pm 3$ & $87 \pm 3$ & $36 \%$ \\
\hline 3391 & 1 fem & acclusion & & & & \\
\hline Mean \pm sem & & $246 \pm 44$ & $114 \pm 38$ & $173 \pm 41$ & $132 \pm 41$ & $27 \pm 5 \%$ \\
\hline
\end{tabular}

$(\mu \mathrm{m}$, mean $\pm \mathrm{sem}) ; \mathrm{r} / \mathrm{fem}=$ right/left femoral artery; I car = left carotid artery; neointima/media $=$ neointima/media between stent fibres; neointima/media stent $=$ neointima/media on top of stent fibres. 
that the stiffness of the polyester stent construction is similar to that of a stainless steel stent.

The mounting- and release-experiments, however, suggest hysteresis-like behaviour that decreases the radial pressure of the polyester device at implantation. This hysteresis-like behaviour is partly attributable to the braided construction, and is partly a feature of the polymer material itself. The initial loss of radial pressure may even be an advantage, as one of the most likely causes of extensive neointimal proliferation and restenosis is the acute barotrauma to the vessel wall [14]. The employment of polymer stents may reduce acute vessel wall damage, because of a slower build-up of radial pressure and, consequently, a softer settling of the polymer stent against the arterial wall. In addition, some polymer materials may simulate the stressstrain response of a blood vessel much closer than metal. So, in contrast to metallic, self-expanding stents, which are associated with an increased risk of restenosis when more than $0.7 \mathrm{~mm}$ oversized compared to the target vessel diameter [15], this polyester stent has to be selected having a considerably larger ( $2 \mathrm{~mm}, 60 \%$ ) nominal diameter to effectively splint the artery.

\section{In vivo implantations}

Implantation of the polyester stents itself was uneventful, although two stents were eventually found in the aortic bifurcation, most likely due to incorrect placement by the prototype delivery-system, although stent migration as a cause cannot be excluded. After four weeks five of the six properly placed stents were patent, with only limited neointimal proliferation. These results are considerably better than those reported for an other polyester stent [16]. At least two factors may be important in the interpretation of this difference in results: a. in the study reported by Murphy et al. stents with an unconstrained diameter of only $3 \mathrm{~mm}$ (contrary to our stents of $5.3 \mathrm{~mm}$ ) were used in the coronary circulation, which may explain the $100 \%$ occlusion rate, most likely due to thrombosis, since polyester can activate platelets systemically [17], and b. the composition of the polyester was probably not identical.

\section{Angiography}

Quantitative angiographic assessment showed no increase in luminal diameter after stenting (diameter change $-0.1 \pm 0.2 \mathrm{~mm}$, i.e. no oversizing) The fact that the arteries were not overstretched may have contributed to the high patency rate. In addition, the angiographic data verified that the considerable hysteresis-like behaviour, found in vitro, also occurred in vivo. On the other hand, the stents proved firm enough to resist vasospasm, which is always present to some extent after the placement of stents in porcine arteries.

\section{Morphology and intravascular ultrasound}

Microscopical examination showed a limited amount of neointimal proliferation in the polyester stents. The mean neointimal hyperplasia on top of the stent fibres $(114 \pm 38 \mu \mathrm{m})$ compared well with the mean change in angiographically measured lumen diameter. On a qualitative basis this also applied to the results obtained with intravascular ultrasound. The number of observations (only 5 stents) and the range of values did not allow a quantitative relation of the results obtained by the three imaging modalities. A high accuracy and precision of quantitative angiography to measure the exact stenosis dimentions has been demonstrated [18]. Larger series of observations are needed to assess the accuracy of intravascular ultrasound analysis of the hyperplastic response after stent implantation.

The present study shows the feasibility of the arterial implantation of polyester stents. The stents are not radiopaque during fluoroscopy, but can easily be visualized by intravascular ultrasound. Intravascular ultrasound may therefore prove a useful technique for the assessment of correct polymer stent placement and may provide us with serial observations of the vessel wall healing process poststenting. 


\section{References}

1. Dotter CT. Transluminally-placed coilspring endarterial tube grafts. Long-term patency in the canine popliteal artery. Invest Radiol 1969; 4: 329-32.

2. Sigwart U, Puel J, Mirkovitch V et al. Intravascular stent to prevent occlusion and restenosis after transluminal angioplasty. N Engl J Med 1987; 316: 701-6.

3. Serruys PW, Strauss BH, Beatt KJ et al. Angiographic follow-up after placement of a self-expanding coronary-artery stent. N Engl J Med 1991; 324: 13-7.

4. Schatz RA, Baim DS, Leon Met al. Clinical experience with the Palmaz-Schatz coronary stent. Initial results of a multicenter study. Circulation 1991; 83: 148-61.

5. Roubin GS, Cannon AD, Agrawal SK et al. Intracoronary stenting for acute and threatened closure complicating percutaneous transluminal coronary angioplasty. Circulation 1992; 85: 916-27.

6. Van der Giessen WJ, Serruys PW, Van Beusekom HMM et al. Coronary stenting with a new, radiopaque, balloon-expandable endoprosthesis in pigs. Circulation 1991; 83: 178898.

7. De Jaegere PP, Serruys PW, Bertrand ME et al. Wiktor stent implantation in patients with restenosis following balloon angioplasty of a native coronary artery. Am J Cardiol 1992; 69: 598-602.

8. Van der Giessen WJ, Van Beusekom HMM, Van Houten R, Van Woerkens LJ, Verdouw PD Serruys PW. Coronary stenting with polymer coated and not coated Wallstent selfexpanding endoprostheses in pigs. Coronary Artery Dis 1992; 3: 631-40.

9. Murphy JG, Schwartz RS, Huber KC, Holmes DR. Polymeric stents: modern alchemy or the future. J Invas Cardiol 1991; 3: 144-8.

10. Van der Giessen WJ, Serruys PW, Van Woerkens LJ et al.
Arterial stenting with self-expandable and balloon-expandable endoprostheses. Int J Cardiac Imag 1990; 5: 163-71.

11. Reiber JHC, Serruys PW, Kooijman CJ et al. Assessment of short-, medium- and long-term variations in arterial dimensions from computer-assisted quantitation of coronary cineangiograms. Circulation 1985; $71: 280-8$.

12. Gussenhoven EJ, Frietman PAV, The SHK et al. Assessment of medial thinning in atherosclerosis by intravascular ultrasound. Am J Cardiol 1991; 68: 1625-32.

13. Polimeni PI, Cunningham P, Otten MD, McCrea D. Morphometric quantification of atherosclerotic plaques by computer-assisted image-analysis of histographs. Comp Biomed Res 1987; 20: 113-24.

14. Schwartz RS, Murphy JG, Edwards WD et al. Restenosis after balloon angioplasty: a practical proliferative model in porcine coronary arteries. Circulation 1990; 82: 2190-200.

15. Strauss BH, Serruys $P W$, de Scheerder IK et al. Relative risk analysis of angiographic predictors of restenosis with the coronary Wallstent. Circulation 1991; 84: 1636-43.

16. Murphy JG, Schwartz RS, Edwards WD et al. Experimental coronary artery stenosis induced by polymer (PET) implantation in domestic swine [abstr]. J Am Coll Cardiol 1991; 17 (6 Suppl B): 195B.

17. Schoenfeld NA, Connolly R, Ramberg Ket al. The systemic activation of platelets by Dacron grafts. Surg Gynecol Obstet $1988 ; 166: 454-7$.

18. Haase J, Di Mario C, Slager CJ et al. In vivo validation of on-line and off-line geometric coronary measurements using insertion of stenosis phantoms in porcine coronary arteries. Cath Cardiovasc Diag 1992; 27: 16-27.

19. Serruys PW, Strauss BH, Van Beusekom HM, Van der Giessen WJ. Stenting of coronary arteries: has a modern Pandora's Box been opened? J Am Coll Cardiol 1991; 17 : 143B-54B. 